1 Universidade Federal da Bahia (UFBA), Instituto de Saúde Coletiva, Programa de Pós-graduação em Saúde Coletiva - Salvador (BA), Brasil.

jamillyss@hotmail.com

2 Universidade Federal da Bahia (UFBA), Instituto de Saúde Coletiva, Programa de Pós-graduação em Saúde Coletiva - Salvador (BA), Brasil.

carment@ufba.br

\section{Política de saúde no Brasil: produção científica 1988-2014}

\author{
Health policy in Brazil: scientific production 1988-2014
}

Jamilli Silva Santos ${ }^{\mathbf{1}}$, Carmen Fontes Teixeira ${ }^{2}$

RESUMO O objetivo deste trabalho é mapear a produção científica em política de saúde no Brasil, na base SciELO, no período 1988-2014. Foram identificados 769 artigos, classificados em três grupos: a) análises políticas em saúde (10,2\%) com predominância de estudos que analisam a reforma sanitária brasileira; b) estudos sobre financiamento, gestão, organização e infraestrutura do sistema de saúde (28,8\%); c) estudos sobre análises de políticas de saúde específicas (49\%). Constatou-se aumento da quantidade de publicações ao longo do tempo e concentração de estudos no último grupo, evidenciando a progressiva substituição de análises do processo político mais geral por estudos de políticas específicas.

PALAVRAS-CHAVE Política de saúde; Sistema Único de Saúde; Reforma dos serviços de saúde; Atividades científicas e tecnológicas.

ABSTRACT The aim of this study is to map the scientific production in health policy in Brazil, at the SciELO base, in the period 1988-2014. 769 articles, divided into three groups, were identified: a) policy analysis in health (10.2\%) with a predominance of studies that analyze the Brazilian health reform; b) studies on financing, management, organization and infrastructure of the health system (28.8\%); c) studies about analysis of specific health policies (49\%). It was found an increase in the number of publications over time and concentration of studies in the last group, highlighting the progressive replacement of analysis of the more general political process by studies of specific policies.

KEYWORDS Health policy; Unified Health System; Health care reform; Scientific and technical activities. 


\section{Introdução}

A revisão do estado da arte na área de Política, Planejamento e Gestão em Saúde (PPGS) é primordial para o desenvolvimento de estudos científicos, pois permite conhecer tendências, lacunas e desafios que se colocam para os pesquisadores, estudantes e gestores do sistema de saúde (LEVCOVITZ ET AL., 2003; PAIM; TEIXEIRA, 2006; TEIXEIRA ET AL., 2014).

Especificamente no que diz respeito a estudos sobre política de saúde, tomou-se como ponto de partida o conceito proposto por Paim (2003), segundo o qual 'política de saúde’ é a resposta social (ação ou omissão) do Estado aos problemas e necessidades de saúde da população, contemplando, portanto, a intervenção sobre a produção, distribuição, gestão e regulação de bens e serviços que afetam a saúde, inclusive o ambiente. Desse modo, abrange as questões relativas ao 'poder' em saúde (natureza, estrutura, relações, distribuição e lutas), bem como as que se referem ao estabelecimento de 'diretrizes, planos e programas' de saúde. Ou seja, contempla tanto os planos de ação governamental (KINGDON, 2011) quanto a análise das relações de poder em saúde (TESTA, 1995).

Enquanto disciplina acadêmica, política de saúde inclui estudos sobre o papel do Estado, as relações Estado-sociedade, movimentos sociais em saúde, as relações entre políticas de saúde e políticas econômicas e sociais (PAIM, 2003; LEVCOVITZ ET AL., 2003) e outros aspectos relativos à análise dos processos políticos que ocorrem em diversos espaços sociais. Também abarca estudos que tratam da formulação e implementação de políticas específicas no âmbito governamental, abordando, por exemplo, a conformação da agenda política em saúde, a elaboração de propostas de intervenção sobre problemas de grupos populacionais específicos, bem como a gestão, implantação e avaliação de planos, programas e projetos.

O presente estudo tem por objetivo mapear a produção científica em política de saúde no Brasil no período pós-constituinte (1988-2014), considerando que, a partir de 1988, com a incorporação do direito à saúde na Constituição Federal, fruto de um amplo processo de Reforma Sanitária Brasileira (RSB) (PAIM, 2008), tem ocorrido um aumento do interesse dos pesquisadores da área em investigar diversos aspectos do processo político em saúde (TEIXEIRA ET AL., 2014), partindo da seguinte pergunta de investigação: quais os principais temas e questões abordadas nos estudos realizados nesse período? Quais as características da evolução temporal dessa produção? É possível identificar alguma correlação entre as temáticas abordadas, o processo de RSB e a construção do Sistema Único de Saúde (SUS) nesse período?

Desse modo, definiram-se como objetivos específicos: a) identificar os principais temas e questões abordadas; b) classificar os estudos que tratam de políticas específicas e os que tratam do processo político em saúde; c) analisar a correlação entre os estudos realizados e o processo político em saúde no período 1988-2014.

\section{Estratégia metodológica}

Trata-se de estudo de revisão sistemática da literatura mediante a aplicação de métodos explícitos e sistematizados de busca, apreciação crítica e síntese da informação selecionada (SAMPAIO; MANCINI, 2007). A estratégia para mapear a produção científica sobre a temática de política de saúde foi concebida a partir de uma revisão bibliográfica que permitiu identificar três trabalhos de relevo no estudo de tendências da produção científica brasileira desde a década de 1970 (LEVCOVITZ ET AL., 2003; PAIM; TEIXEIRA, 2006; TEIXEIRA ET AL., 2014). A análise da tipologia das áreas e subáreas temáticas construídas em cada um desses estudos permitiu construir uma quarta tipologia que constituiu o referencial para a busca e seleção 
de documentos na base de dados SciELO (Scientific Electronic Library Online).

O ponto de partida para o mapeamento da produção científica implicou, em primeiro lugar, na definição dos descritores de assunto, com consulta a especialistas na área, bem como análise dos descritores disponíveis nos trabalhos anteriores, aliados ao recurso ao vocabulário controlado do DeCS (Descritores em Ciências da Saúde) e à própria estratégia de indexação na SciELO. Mediante utilização dos descritores 'política de saúde', 'Sistema Único de Saúde' e 'direito à saúde', foram então acessados os artigos na SciELO homepage do Brasil por abrigar em seu acervo a coleção recente de periódicos científicos brasileiros.

Foram identificados 824 artigos, dos quais foram extraídos 55 que apareceram repetidos em função do uso de três descritores correlatos. Com isso, obteve-se um total de 769 artigos publicados no período 1988 a 2014, os quais foram submetidos a uma reclassificação a partir da leitura do título do estudo, levando-se em conta a especificidade do objeto de estudo conforme a tipologia descrita no quadro 1. Foram incluídos no primeiro grupo os estudos que tratam de análise política em saúde (politics), em um segundo conjunto, os estudos que abordam a dinâmica política em torno dos diversos componentes do sistema de saúde - o financiamento; gestão/participação e controle social; modelos de atenção; recursos humanos; informação, ciência e tecnologia em saúde -, e em um terceiro, os estudos que tratam de análises de políticas de saúde específicas (policy).

Quadro 1. Componentes das sete dimensões do ACIC

\begin{tabular}{l|l}
\hline \multicolumn{1}{c|}{ Áreas } & \multicolumn{1}{c}{ Definição } \\
\hline 1. Análise política em saúde & $\begin{array}{l}\text { Política de saúde em uma perspectiva internacional; Reforma Sanitária } \\
\text { Brasileira; processo de construção do SUS; relações entre o público e o } \\
\text { privado (SUS-Sams). }\end{array}$ \\
\hline 2. Componentes do sistema de saúde & $\begin{array}{l}\text { Financiamento da saúde (volume de recursos, formas de distribuição, } \\
\text { custos etc.); Gestão de sistemas de saúde (descentralização, regionaliza- } \\
\text { cãa, modalidades alternativas de gestão); Participação e controle social; } \\
\text { Modelos de atenção em saúde; Recursos humanos em saúde; Ciência, } \\
\text { tecnologia e inovação. }\end{array}$ \\
\hline $\begin{array}{l}\text { 3. Análise de políticas de saúde especí- } \\
\text { ficas }\end{array}$ & $\begin{array}{l}\text { Políticas voltadas a grupos populacionais específicos (mulher, criança, } \\
\text { idoso, trabalhador etc.) ou ao enfrentamento de problemas específicos } \\
\text { (Aids, dengue, hipertensão arterial, tuberculose etc.). }\end{array}$ \\
\hline
\end{tabular}

Fonte: Elaboração própria.

Uma vez realizada essa reclassificação, tomou-se cada um dos três conjuntos de artigos e procedeu-se a uma subdivisão temática, buscando-se identificar os subtemas abordados pelos autores. Com isso, foram construídas tabelas que apontam o mosaico de questões selecionadas pelos diversos autores. Nessa perspectiva, os estudos que tratam de análise política ao nível macro (internacional ou nacional) foram reagrupados segundo o tema abordado. $\mathrm{O}$ mesmo foi feito com relação aos estudos sobre componentes do sistema de saúde (KLECZKOWSKI; ROEMER; VAND DER WERFF, 1984), reclassificados segundo o componente analisado. Por fim, também foram reagrupados os estudos que tratam de análise de políticas específicas, sendo que, nesse caso, adotou-se uma classificação derivada da pesquisa realizada no site do Ministério da Saúde que permitiu a identificação de 'políticas' elaboradas e formalizadas em documentos institucionais no período 2003-2014. 


\section{Resultados e discussão}

A produção científica sobre política de saúde registrada no SciELO no período 1988-2014 totaliza, conforme explicitado anteriormente, 769 artigos, distribuídos, segundo a tipologia adotada neste trabalho, em três grupos. Dos estudos, 10,2\% (78) contêm análises do processo político em saúde, enquanto $28,8 \%$ abordam aspectos referentes aos componentes do sistema de saúde. Quase metade dos trabalhos trata de análises de políticas de saúde específicas (49,0\%), e $12,0 \%$ dos artigos correspondem a estudos que não se enquadram nos grupos anteriormente descritos (tabela 1).

Tabela 1. Distribuição dos artigos segundo objeto de estudo. Brasil, 1988-2014

\begin{tabular}{lcr}
\hline Áreas & N & $\%$ \\
\hline 1. Análise política em saúde & 78 & 10,2 \\
\hline 2. Componentes do sistema de saúde & 221 & 28,8 \\
\hline 3. Análise de políticas de saúde específicas & 377 & 49,0 \\
\hline 4. Outros & 93 & 12,0 \\
\hline Total & 769 & 100,0 \\
\hline Fonte: Elaboração própria. & &
\end{tabular}

\section{a) Distribuição dos estudos de aná- lise política em saúde por subárea temática}

O primeiro trabalho entre os 78 incluídos nessa área temática foi publicado em 1988. Durante toda a década de 1990, observou-se apenas a publicação de um trabalho por ano, sendo que, a partir do ano 2000 , esse número cresceu, de modo que, no período compreendido entre 2008 e 2014, encontra-se mais da metade das publicações (52,0 \%), o que talvez evidencie um aumento do número de pesquisadores da área que adotam uma perspectiva de análise abrangente acerca do processo político em saúde no País.

De fato, a redistribuição dos 78 artigos incluídos neste grupo por subárea temática (tabela 2) evidencia predominância de estudos acerca da política de saúde no Brasil (53,8\%), especialmente do processo de RSB e construção do SUS, verificando-se também estudos que abordam a política de saúde em uma perspectiva internacional (20,5\%) e pequena percentagem de estudos acerca da relação entre o SUS e o Sistema de Assistência Médica Suplementar (Sams). O restante dos trabalhos (21,8\%) trata de análise política em saúde em uma perspectiva localizada (estudos de casos) e discussão de elementos teóricos para a análise de políticas sociais.

Esses achados evidenciam o interesse dos pesquisadores da área em acompanhar e analisar o processo de reforma do sistema de saúde ao longo dos últimos 27 anos, chamando atenção a grande quantidade de trabalhos que se focam sobre os processos que incidem sobre o sistema público (SUS), aos quais se agrega um interesse crescente na problematização das relações entre o público e o privado, o que, de certo modo, reflete as tensões e contradições que perpassam o processo de implementação do SUS no Brasil nesse período (PAIM ET AL., 2011; PAIM, 2013A; TEIXEIRA ET AL., 2014). 
Tabela 2. Estudos de análise política em saúde por subárea temática. Brasil, 1988-2014

\begin{tabular}{lrr}
\hline \multicolumn{1}{c}{ Subárea Temática } & Artigos & \\
\cline { 2 - 3 } & N & $\%$ \\
\hline 1. Políticas de saúde em uma perspectiva internacional & 16 & 20,5 \\
\hline 2. Política de saúde no Brasil: RSB/SUS (Geral) & 42 & 53,8 \\
\hline 3. Relação entre o público e o privado (SUS/Sams) & 3 & 3,9 \\
\hline 4. Outros & 17 & 21,8 \\
\hline Total & 78 & 100
\end{tabular}

Fonte: Elaboração própria.

\section{b) Distribuição dos estudos sobre o SUS segundo os componentes do sistema}

Foram encontrados 221 artigos sobre os componentes do sistema de saúde (tabela 3). A subárea temática mais frequente foi a de gestão de sistemas e serviços de saúde
(33,4\%), seguida das subáreas modelos de atenção à saúde (16,7\%), recursos humanos em saúde e ciência, tecnologia e inovação com 15,0 \% das publicações cada uma. As subáreas participação e controle social $(11,8 \%)$ e financiamento da saúde apresentaram os menores números de trabalhos $(8,1 \%)$.

Tabela 3. Estudos sobre SUS segundo componentes do sistema. Brasil, 1988-2014

\begin{tabular}{llr}
\hline \multicolumn{1}{c}{ Subárea Temática } & Artigos & \\
\cline { 2 - 3 } & $\mathbf{N}$ & $\%$ \\
\hline a) Financiamento da saúde & 18 & 8,1 \\
\hline b) Gestão de sistemas e serviços de saúde & 74 & 33,4 \\
\hline c) Modelos de atenção à saúde & 37 & 16,7 \\
\hline d) Recursos humanos em saúde & 33 & 15,0 \\
\hline e) Ciência, tecnologia e inovação & 33 & 15,0 \\
\hline f) Participação e controle social & 26 & 11,8 \\
\hline Total & 221 & 100,0 \\
\hline
\end{tabular}

Fonte: Elaboração própria.

Essa distribuição revela o maior interesse dos pesquisadores com a problemática da gestão do sistema, abordada sob diversos ângulos, inclusive na perspectiva da chamada 'gestão participativa', o que pode explicar o pequeno número de trabalhos que abordam especificamente a 'participação e controle social'. Chama a atenção, entretanto, o reduzido número de trabalhos sobre financiamento da saúde, um dos temas mais importantes na agenda política do setor, o que pode ter decorrido de certo viés na captura dos artigos, uma vez que não foi utilizado um descritor específico sobre esse tema.

\section{c) Distribuição dos estudos sobre políticas específicas do Ministério da Saúde}

Ao considerar que o Ministério da Saúde contempla em seu organograma responsabilidades definidas sobre políticas específicas, 
os 377 artigos referentes a políticas de saúde foram distribuídos segundo estas áreas (BRASIL, 2010), a saber: atenção à saúde; gestão do trabalho e educação da saúde; ciência, tecnologia e insumos estratégicos; gestão estratégica e participativa; vigilância em saúde e saúde indígena (tabela 4).

A área que apresenta maior número de trabalhos é a de atenção à saúde (59,6\%), seguida das áreas de gestão do trabalho e educação da saúde (19,9\%), ciência, tecnologia e insumos estratégicos (10,6\%), vigilância em saúde (7,8\%), gestão estratégica e participativa $(1,6 \%)$ e, por fim, com o menor escore, a área de saúde indígena $(0,5 \%)$.

$\mathrm{Na}$ área de atenção à saúde, destaca-se a predominância das publicações nas subáreas da 'atenção básica' (19,9\%) e 'atenção especializada e temática' (19,2\%). Na subárea atenção básica, a maioria dos estudos trata especificamente dessa política, provavelmente pela importância concedida à estratégia de saúde da família, abordada sob diversos ângulos (TEIXEIRA; SOLLA, 2006). Em um distante segundo lugar, estão os estudos sobre a política de saúde bucal, o que também parece estar vinculado à incorporação dessas ações no âmbito da atenção básica, principalmente nos últimos 12 anos (SOUZA; RONCALLI, 2007).

Na subárea 'ações programáticas e estratégicas', há predominância de estudos sobre a Política Nacional de Atenção Integral à Saúde da Mulher (6,9\%), e em segundo lugar, sobre a Política Nacional de Atenção Integral à Saúde da Criança e Aleitamento Materno $(4,8 \%)$, o que pode refletir a prioridade tradicionalmente atribuída a esses dois grupos na atenção primária à saúde, embora o modelo empregado preconize uma abordagem familiar (SANTOS NETO, 2008).

Observa-se na subárea 'regulação, avaliação e controle de sistemas' o menor número de estudos $(0,8 \%)$, os quais abordavam aspectos dos sistemas de informação e controle de sistemas e serviços em detrimento da regulação e avaliação, planejamento e programação das ações, não abordados nos estudos.
Na subárea 'atenção hospitalar e urgência' $(3,2 \%)$, verifica-se discreta concentração de estudos acerca da Política Nacional de Atenção Hospitalar (1,3\%), tema que parece estar sendo abordado mais em função de a relevância da atenção hospitalar ser o componente do modelo assistencial responsável pela maior absorção de recursos (CARVALHO, 2007) do que do ponto de vista da política governamental. Já a existência de estudos sobre a Política Nacional de Transplantes de Órgãos e Tecidos (1,1\%), ainda que em número reduzido, sinaliza a importância do Programa de Doação, Captação e Transplante de Órgãos e Tecidos no Brasil, o qual realiza todos os tipos de transplantes com financiamento do SUS, incluindo oferta gratuita da medicação necessária após a realização das cirurgias, fazendo com que o País possua atualmente o maior programa público de transplantes do mundo (MARINHO, 2006).

A Política Nacional de Humanização, também componente da área de 'atenção à saúde', foi abordada em $4 \%$ dos estudos, percentual esse superior à subárea anteriormente citada, tal achado demonstrando a relevância que vem sendo atribuída à mudança nos modos de gerir e cuidar em saúde, que motivou a criação da Política Nacional de Humanização em 2003 (PASCHE; PASSOS; HENNINGTON, 2011).

Segunda área com maior número de publicações (19,9\%), a 'gestão do trabalho e educação da saúde' concentra-se em estudos da gestão da educação na saúde (17,2\%) em comparação à Política Nacional de Educação Permanente em Saúde, que foi estudada em apenas $2,7 \%$ dos trabalhos. Tal achado sinaliza maior dedicação dos estudos relacionados à formação profissional em saúde em detrimento da educação permanente dos profissionais e trabalhadores do setor, tema só mais recentemente priorizado na agenda política do sistema.

$\mathrm{Na}$ área de ciência, tecnologia e insumos estratégicos, foi encontrada percentagem quatro vezes superior de estudos sobre a Política Nacional de Assistência Farmacêutica 
(8,5\%), o que indica a relevância que a assistência farmacêutica tem assumido no SUS nos últimos anos na tentativa de melhorar o acesso dos brasileiros aos fármacos de que necessitam, podendo-se citar iniciativas como o Programa de Farmácia Popular, criado em 2004, e sua expansão para a rede privada em 2006 (VIEIRA, 2010).

Contudo, o acesso ainda é limitado, motivando inclusive onda crescente de ações judiciais para fornecimento de medicamentos e produtos, que integram o contemporâneo fenômeno da "judicialização da saúde" (PEPE ET AL., 2010, p. 2406). O pequeno número de estudos sobre Política Nacional de Ciência, Tecnologia e Inovação em Saúde (2,1\%), por sua vez, pode estar refletindo o mesmo processo indicado para a relação gestão da educação na saúde/educação permanente, ou seja, esta temática só ganhou relevância da agenda política nos últimos 12 anos, o que provavelmente vem estimulando a realização de estudos acerca da incorporação de tecnologias e inovações no SUS.

Tabela 4. Distribuição dos artigos segundo políticas de saúde específicas. Brasil, 1988-2014

Artigos por Áreas, Subáreas Temáticas e Políticas

$\mathrm{N}$

$\%$

\begin{tabular}{lrr}
\hline 1. Atenção à Saúde & $\mathbf{2 2 5}$ & $\mathbf{5 9 , 6}$ \\
\hline 1.1 Atenção Básica & $\mathbf{7 5}$ & $\mathbf{1 9 , 9}$ \\
\hline 1.1.1 Política Nacional de Atenção Básica & 50 & 13,3 \\
\hline 1.1.2 Política Nacional de Saúde Bucal & 13 & 3,4 \\
\hline 1.1.3 Política Nacional de Alimentação e Nutrição & 02 & 0,5 \\
\hline $\begin{array}{l}\text { 1.1.4 Política Nacional de Práticas Integrativas e Comple- } \\
\text { mentares }\end{array}$ & 10 & 2,7 \\
\hline
\end{tabular}

\begin{tabular}{|c|c|c|}
\hline 1.2 Atenção Especializada e Temática & 73 & 19,2 \\
\hline 1.2.1 Política Nacional de Saúde da Pessoa Idosa & 05 & 1,3 \\
\hline 1.2.2 Política Nacional de Média e Alta Complexidade & 19 & 5,0 \\
\hline $\begin{array}{l}\text { 1.2.3 Política/Programa de Atenção às Pessoas com Doen- } \\
\text { ças Crônicas }\end{array}$ & 13 & 3,4 \\
\hline 1.2.4 Política Nacional de Saúde Mental & 27 & 7,2 \\
\hline $\begin{array}{l}\text { 1.2.5 Política de Atenção Integral aos Usuários de Álcool e } \\
\text { Outras Drogas }\end{array}$ & 04 & 1,0 \\
\hline $\begin{array}{l}\text { 1.2.6 Política Nacional de Atenção Integral à Saúde do } \\
\text { Homem }\end{array}$ & 05 & 1,3 \\
\hline 1.3 Ac̣ões Programáticas e Estratégicas & 47 & 12,5 \\
\hline $\begin{array}{l}\text { 1.3.1 Política Nacional de Atenção Integral à Saúde das } \\
\text { Pessoas Privadas de Liberdade no Sistema Prisional }\end{array}$ & 00 & 0,0 \\
\hline $\begin{array}{l}\text { 1.3.2 Política Nacional de Atenção Integral à Saúde da } \\
\text { Mulher }\end{array}$ & 26 & 6,9 \\
\hline $\begin{array}{l}\text { 1.3.3 Política Nacional de Atenção Integral à Saúde da } \\
\text { Criança e Aleitamento Materno }\end{array}$ & 18 & 4,8 \\
\hline $\begin{array}{l}\text { 1.3.4 Política Nacional de Atenção Integral à Saúde do } \\
\text { Adolescente }\end{array}$ & 01 & 0,3 \\
\hline 1.3.5 Política Nacional de Saúde da Pessoa com Deficiência & 02 & 0,5 \\
\hline 1.4 Regulação, Avaliação e Controle de Sistemas & 03 & 0,8 \\
\hline 1.4.1 Sistemas de Informação & 02 & 0,5 \\
\hline
\end{tabular}


Tabela 4. (cont.)

\begin{tabular}{|c|c|c|}
\hline 1.4.2 Controle de Serviços e Sistemas & 01 & 0,3 \\
\hline 1.4.3 Regulação e Avaliação & 00 & 0,0 \\
\hline 1.4.4 Planejamento e Programação das Ações em Saúde & 00 & 0,0 \\
\hline 1.5 Atenção Hospitalar e Urgência & 12 & 3,2 \\
\hline $\begin{array}{l}\text { 1.5.1 Política Nacional de Sangue, Componentes e Hemo- } \\
\text { derivados }\end{array}$ & 01 & 0,3 \\
\hline 1.5.2 Rede de Atenção às Urgências e Emergências & 02 & 0,5 \\
\hline 1.5.3 Política Nacional de Transplantes de Órgãos e Tecidos & 04 & 1,1 \\
\hline 1.5.4 Política Nacional de Atenção Hospitalar & 05 & 1,3 \\
\hline 1.6 Política Nacional de Humanização & 15 & 4,0 \\
\hline 2. Gestão do Trabalho e Educação na Saúde & 75 & 19,9 \\
\hline 2.1 Gestão da Educação na Saúde & 65 & 17,2 \\
\hline 2.2 Política Nacional de Educação Permanente em Saúde & 10 & 2,7 \\
\hline 3. Ciência, Tecnologia e Insumos Estratégicos & 40 & 10,6 \\
\hline 3.1 Política Nacional de Assistência Farmacêutica & 32 & 8,5 \\
\hline $\begin{array}{l}\text { 3.2 Política Nacional de Ciência, Tecnologia e Inovação em } \\
\text { Saúde }\end{array}$ & 08 & 2,1 \\
\hline 4. Gestão Estratégica e Participativa & 06 & 1,6 \\
\hline $\begin{array}{l}\text { 4.1 Política Nacional de Gestão Estratégica e Participativa } \\
\text { no SUS }\end{array}$ & 02 & 0,5 \\
\hline 4.2 Política Nacional de Educação Popular em Saúde & 01 & 0,3 \\
\hline 4.3 Política Nacional de Saúde Integral da População Negra & 03 & 0,8 \\
\hline $\begin{array}{l}\text { 4.4 Política Nacional de Saúde Integral de Outras Popula- } \\
\text { cões (Lésbicas, Gays, Bissexuais, Travestis e Transexuais; } \\
\text { Campo e Floresta; em Situação de rua e Cigana) }\end{array}$ & 00 & 0,0 \\
\hline 5. Vigilância em Saúde & 29 & 7.8 \\
\hline 5.1 Vigilância Epidemiológica & 07 & 1,9 \\
\hline 5.1.1 Programa Nacional de Controle da Tuberculose & 03 & 0,8 \\
\hline $\begin{array}{l}\text { 5.1.2 Programa Nacional de Imunizações, Controle da Han- } \\
\text { seníase, da Dengue, da Malária }\end{array}$ & 00 & 0,0 \\
\hline 5.1.3 Controle de Chagas e Esquistossomose & 04 & 1,1 \\
\hline 5.2 Análise de Situação de Saúde & 07 & 1,9 \\
\hline 5.2.1 Política Nacional de Promoção da Saúde & 07 & 1,9 \\
\hline $\begin{array}{l}\text { 5.3 Vigilância, Prevenção e Controle das Doenças Sexual- } \\
\text { mente Transmissíveis, Síndrome da Imunodeficiência } \\
\text { Adquirida e Hepatites Virais }\end{array}$ & 08 & 2,1 \\
\hline $\begin{array}{l}\text { 5.3.1 Política Nacional de Prevenção de DST/HIV/Aids e } \\
\text { Hepatites Virais }\end{array}$ & 08 & 2,1 \\
\hline 5.4 Vigilância em Saúde Ambiental e do Trabalhador & 07 & 1,9 \\
\hline 5.4.1 Política Nacional de Saúde Ambiental & 01 & 0,3 \\
\hline 5.4.2 Política Nacional de Saúde do Trabalhador & 06 & 1,6 \\
\hline
\end{tabular}


Tabela 4. (cont.)

\begin{tabular}{lcc}
\hline 6. Saúde Indígena & $\mathbf{0 2}$ & $\mathbf{0 , 5}$ \\
\hline $\begin{array}{l}\text { 6.1 Política Nacional de Atenção à Saúde dos Povos Indíge- } \\
\text { nas }\end{array}$ & 02 & 0,5 \\
\hline Total & $\mathbf{3 7 7}$ & $\mathbf{1 0 0 , 0}$ \\
\hline
\end{tabular}

Fonte: Elaboração própria.

$\mathrm{Na}$ área de 'gestão estratégica e participativa', predominam estudos acerca da Política Nacional de Saúde Integral da População Negra em detrimento de outros grupos $(0,8 \%)$, o que pode estar relacionado com a representatividade e consolidação do Movimento Social Negro (LIMA, 2010). Chama a atenção o fato de ter aparecido apenas um estudo sobre a Política Nacional de Educação Popular em Saúde e não aparecerem publicações sobre várias outras políticas que têm sido formuladas mais recentemente, como é o caso da Política Nacional de Saúde Integral de Outras Populações (lésbicas, gays, bissexuais, travestis e transexuais; campo e floresta; em situação de rua e cigana).

$\mathrm{Na}$ área de 'vigilância em saúde', estudos sobre a Política Nacional de Prevenção de DST/HIV/Aids e hepatites virais foram os mais frequentes $(2,1 \%)$, sugerindo a relevância atribuída à temática no País que conta com movimento social organizado em torno do tema e com um programa que, embora tenha dividido opiniões quanto à sua efetividade, destaca-se no mundo por oferecer tratamento antirretroviral gratuito (VILLARINHO ET AL., 2013). Em segundo lugar, aparecem os estudos acerca da Política Nacional de Promoção da Saúde (1,9\%), tema que tem sido abordado por vários pesquisadores, sendo que a divulgação dos resultados desses estudos não tem se dado por meio de artigos científicos, e sim por intermédio de livros e capítulos de livros (TEIXEIRA, 2006; PELLEGRINI FILHO; BUSS; ESPERIDIÃO, 2014). Foi encontrado também um único estudo sobre Política Nacional de Saúde Ambiental, e não houve publicações sobre os Programas
Nacionais de Imunizações e Controle da Hanseníase, da Dengue e da Malária.

Por fim, a área de saúde indígena foi contemplada com apenas $0,5 \%$ das publicações. Embora corresponda à parcela numericamente pouco expressiva da população, os índios têm grande importância histórico-cultural para o País, o que poderia motivar parcela mais expressiva de estudos dedicados às suas diversas demandas, muito específicas, relativas à sua sobrevivência física e cultural diante da acelerada e complexa transformação social a que foram submetidos (CHAVES; CARDOSO; ALMEIDA, 2006).

\section{Considerações finais}

O mapeamento da produção científica sobre política de saúde no período 1988-2014 trata de uma primeira aproximação que será desdobrada em estudos posteriores que identifiquem as abordagens teóricas utilizadas pelos diversos autores. O estudo, entretanto, revela algumas características da prática de pesquisa que vem sendo desenvolvida na área de PPGS.

Em primeiro lugar, observa-se uma tendência ao aumento da quantidade de artigos publicados ao longo do período, o que certamente reflete o aumento exponencial de grupos de pesquisa nessa área, os quais somavam, em abril de 2015, cerca de 80 grupos registrados na plataforma de grupos de pesquisa do Conselho Nacional de Desenvolvimento Científico e Tecnológico (CNPq). Tal fato pode estar ligado, inclusive, ao aumento do número de programas de 
cursos de pós-graduação na área de saúde coletiva.

Em segundo lugar, chama atenção a mudança que vem se verificando na distribuição desses trabalhos por área temática, o que evidencia uma mudança na seleção dos objetos de investigação e, provavelmente, uma diversificação de abordagens teórico-metodológicas aos diversos temas. De fato, no levantamento feito dez anos atrás (PAIM; TEIXEIRA, 2006), observou-se que a maioria dos estudos sobre política de saúde realizados até o início da década de 1990 apresentava uma perspectiva macro, utilizando categorias extraídas da abordagem marxista, tomando como referencial as relações Estado-sociedade, determinantes dos processos políticos na área de saúde. Paulatinamente, foram surgindo investigações sobre políticas, instituições e práticas de saúde, valorizando-se, também, estudos de avaliação em saúde, que incluem avaliação dos efeitos de políticas específicas.

Essa tendência parece ter se intensificado nos últimos anos, uma vez que o mapeamento do conjunto da produção revela a concentração dos estudos na análise de políticas específicas, o que pode estar refletindo a própria fragmentação do processo de formulação e implementação de políticas de saúde no Brasil, com ênfase na elaboração de propostas de intervenção sobre problemas apresentados por grupos populacionais específicos, que ao se organizarem politicamente, passam a atuar em diversos espaços governamentais.

Chama a atenção, portanto, o número relativamente reduzido de estudos sobre as questões do poder em saúde analisado na perspectiva macropolítica, da relação

\section{Referências}

BRASIL. Ministério da Saúde. Regimentos Internos e organogramas básicos do Ministério da Saúde. Ministério da Saúde: Brasília, DF, 2010. entre Estado e as classes sociais no Brasil contemporâneo, especialmente tendo em vista a problematização da sustentabilidade política do processo de RSB e os determinantes das tendências atuais de valorização do mercado de serviços de saúde, expressas tanto na expansão dos planos de saúde e serviços privados quanto na privatização do sistema público (COSTA; BAHIA; SCHEFFER, 2013; SESTELO; SOUZA; BAHIA, 2013; SCHEFFER, 2015).

É possível que isso reflita, indiretamente, a diversidade de concepções acerca da vitalidade do processo de RSB (HOCHMAN, 2013; PAIM, 2013b), com grande parte dos estudiosos se ocupando muito mais dos processos institucionais do que da luta política mais geral para viabilizar a RSB enquanto uma reforma social, uma mudança no "modo de vida" (PAIM, 2008, p. 38).

Isso indica a necessidade de avançar na leitura dos textos completos desses trabalhos, de modo a identificar a filiação dos autores a determinadas correntes de pensamento e a determinadas posições políticas com relação à RSB, tendo como referência o debate político mais geral sobre os rumos da sociedade brasileira no contexto atual e as perspectivas que se desenham em termos de política de saúde. Esse é exatamente o desafio que se está tratando de enfrentar como parte da pesquisa que vem sendo desenvolvida no âmbito do Observatório da Rede de Políticas de Saúde, sediado no Instituto de Saúde Coletiva da Universidade Federal da Bahia, cuja finalidade principal é subsidiar o debate sobre os modos de produzir conhecimento cientificamente fundamentado e politicamente comprometido com o avanço do processo de Reforma Sanitária no País.

CARVALHO, D. M. T. Financiamento da assistência médico-hospitalar no Brasil. Ciênc. saúde coletiva, Rio de Janeiro, v. 12, n. 4, p. 879-892, ago. 2007. 
CHAVES, M. B. G.; CARDOSO, A. M.; ALMEIDA, C. Implementação da política de saúde indígena no pólobase Angra dos Reis. Cad. Saúde Pública, Rio de Janeiro, v. 22 , n. 2 , p. 295-305, fev. 2006.

COSTA, A. M.; BAHIA, L.; SCHEFFER, M. Onde foi parar o sonho do SUS? Le Monde Diplomatique Brasil, v. 69, p. 30-31, 2013.

HOCHMAN, G. Saudades do futuro ou um sistema de saúde em tempos democráticos. Cad. Saúde Pública. Rio de Janeiro, v. 29, n. 10, p. 1927-1953, out. 2013.

KINGDON, J. W. Agendas, alternatives, and public policies. 3. ed. Longman: Washington, 2011.

KLECZKOWSKI, B.; ROEMER, M.; VAN DER WERFF,

A. Sistemas nacionales de salud y su reorientación hacia la salud para todos. OMS Cuadernos Salud Pública, n. 77, 1984.

LEVCOVITZ, E. et al. Produção de conhecimento em política, planejamento e gestão em saúde e políticas de saúde no Brasil (1974-2000). OPAS: Brasília, DF, 2003 (Projeto de desenvolvimento de sistemas de serviços de saúde, 2).

LIMA, M. Desigualdades raciais e políticas públicas: ações afirmativas no governo Lula. Novos estud. CEBRAP, São Paulo, n. 87, p. 77-95, jul. 2010.

MARINHO, A. Um estudo sobre as filas para transplantes no Sistema Único de Saúde brasileiro. Cad. Saúde Pública, Rio de Janeiro, v. 22, n. 10, p. 2229-2239, out. 2006.

PAIM, J. S. A constituição cidadã e os 25 anos do Sistema Único de Saúde. Cad. Saúde Pública, v. 29, n. 10, p. 19271936, 2013a.

Políticas de Saúde no Brasil. In: ROUQUAYROL,

M. Z.; ALMEIDA FILHO, N. Epidemiologia e saúde. 6. ed. Medsi: Rio de Janeiro, 2003, p. 587-603.

Reforma Sanitária Brasileira: contribuição para a compreensão e crítica. Salvador: Edufba: Rio de Janeiro: Fiocruz, 2008.
Reforma Sanitária Brasileira: eppur si muove. Cad. Saúde Pública, Rio de Janeiro, v. 29, n. 10, p. 1952-1953, out., 2013 b.

PAIM, J. S.; TEIXEIRA, C. F. Política, planejamento e gestão em saúde: balanço do estado da arte. Rev. Saúde Pública, São Paulo, v. 40, n. esp., p. 73-78, ago. 2006.

PAIM, J. S. et al. O sistema de saúde brasileiro: história, avanços e desafios. The Lancet, p. 11-31, maio 2011. Disponível em: <http://www6.ensp.fiocruz.br/ repositorio/sites/default/files/arquivos/Sistema\%20 de\%20sa\%C3\%BAde_Celia\%20Almeida_2011.pdf>. Acesso em: 2 dez. 2015.

PASCHE, D. F.; PASSOS, E.; HENNINGTON, E. A. Cinco anos da política nacional de humanização: trajetória de uma política pública. Ciênc. saúde coletiva, Rio de Janeiro, v. 16, n. 11, p. 4541-4548, nov. 2011.

PELLEGRINI FILHO, A.; BUSS, P. M.; ESPERIDIÃO, M. A. Promoção da Saúde e seus fundamentos: deter-minantes sociais da saúde, ação intersetorial e políticas saudáveis. In: PAIM, J. S.; ALMEIDA FILHO, N. (Org.). Saúde coletiva: teoria e prática. Rio de Janeiro: Med Book, 2014. p. 305-326.

PEPE, V. L. E. et al. A judicialização da saúde e os novos desafios da gestão da assistência farmacêutica. Ciênc. saúde coletiva, Rio de Janeiro, v. 15, n. 5, p. 2405-2414, ago. 2010.

SAMPAIO, R. F.; MANCINI, M. C. Estudos de revisão sistemática: um guia para síntese criteriosa da evidência científica. Revista Brasileira de Fisioterapia, São Carlos, v. 11, n. 1, p. 83-89, jan./fev. 2007.

SANTOS NETO, E. T. et al. Políticas de saúde materna no Brasil: os nexos com indicadores de saúde maternoinfantil. Saude soc., São Paulo, v. 17, n. 2, p. 107-119, jun. 2008.

SCHEFFER, M. O capital estrangeiro e a privatização do sistema de saúde brasileiro. Cad. Saúde Pública, Rio de Janeiro, v. 31, n. 4, p. 663-666, abr. 2015.

SESTELO, J. A. F.; SOUZA, L. E. P. F.; BAHIA, L. Saúde 
suplementar no Brasil: abordagens sobre a articulação público/privada na assistência à saúde. Cad. Saúde Pública, Rio de Janeiro, v. 29, n. 5, p. 851-866, maio 2013.

SOUZA, T. M. S.; RONCALLI, A. G. Saúde bucal no Programa Saúde da Família: uma avaliação do modelo assistencial. Cad. Saúde Pública, Rio de Janeiro, v. 23, n. 11, p. 2727-2739, nov. 2007.

TEIXEIRA, C. F. Saúde da família, promoção e vigilância: construindo a integralidade da atenção à saúde no SUS

In: TEIXEIRA, C. F.; SOLLA, J. P. Modelo de atenção à saúde: vigilância e saúde da família. Salvador: Edufba, 2006.

TEIXEIRA, C. F. et al. Produção científica sobre política, planejamento e gestão no campo da saúde coletiva: visão panorâmica. In: PAIM, J. S.; ALMEIDA FILHO, N. Saúde Coletiva: teoria e prática, Rio de Janeiro: Medbook, 2014. p. $585-594$
TEIXEIRA, C. F.; SOLLA, J. P. Modelo de atenção à saúde: vigilância e saúde da família. Salvador: Edufba, 2006.

TESTA, M. Pensamento estratégico e lógica de programação: o caso da saúde. São Paulo: Hucitec: Rio de Janeiro: Abrasco, 1995.

VIEIRA, F. S. Assistência farmacêutica no sistema público de saúde no Brasil. Rev Panam Salud Publica, Washington, v. 27, n. 2, fev. 2010.

VILLARINHO, M. V. et al. Políticas públicas de saúde face à epidemia da AIDS e a assistência às pessoas com a doença. Rev. bras. enferm., Brasília, DF, v. 66, n. 2, p. 271277, abr. 2013.

Recebido para publicação em maio de 2015

Versão final em novembro de 2015

Conflito de interesses: inexistente

Suporte financeiro: não houve 